DRAFT VERSION NOVEMBER 1, 2018

Preprint typeset using L LTEX style emulateapj v. 5/2/11

\title{
OBJECTS APPEAR SMALLER AS THEY RECEDE: HOW PROPER MOTIONS CAN DIRECTLY REVEAL THE COSMIC EXPANSION, PROVIDE GEOMETRIC DISTANCES, AND MEASURE THE HUBBLE CONSTANT
}

\author{
JEREMY DARLING ${ }^{1}$ \\ Draft version November 1, 2018
}

\begin{abstract}
Objects and structures gravitationally decoupled from the Hubble expansion will appear to shrink in angular size as the universe expands. Observations of extragalactic proper motions can thus directly reveal the cosmic expansion. Relatively static structures such as galaxies or galaxy clusters can potentially be used to measure the Hubble constant, and test masses in large scale structures can measure the overdensity. Since recession velocities and angular separations can be precisely measured, apparent proper motions can also provide geometric distance measurements to static structures. The apparent fractional angular compression of static objects is 15 $\mu$ as $\mathrm{yr}^{-1}$ in the local universe; this motion is modulated by the overdensity in dynamic expansion-decoupled structures. We use the Titov et al. quasar proper motion catalog to examine the pairwise proper motion of a sparse network of test masses. Small-separation pairs $(<200 \mathrm{Mpc}$ comoving $)$ are too few to measure the

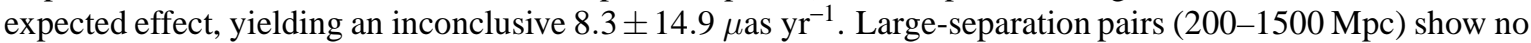
net convergence or divergence for $z<1,-2.7 \pm 3.7 \mu \mathrm{as} \mathrm{yr}^{-1}$, consistent with pure Hubble expansion and significantly inconsistent with static structures, as expected. For all pairs a "null test" gives $-0.36 \pm 0.62 \mu \mathrm{as} \mathrm{yr}^{-1}$, consistent with Hubble expansion, and excludes a static locus at $\sim 5-10 \sigma$ significance for $z \simeq 0.5-2.0$. The observed large-separation pairs provide a reference frame for small-separation pairs that will significantly deviate from the Hubble flow. The current limitation is the number of small-separation objects with precise astrometry, but Gaia will address this and will likely detect the cosmic recession.
\end{abstract}

Subject headings: astrometry — cosmological parameters — cosmology: miscellaneous — cosmology: observations - distance scale - large-scale structure of universe

\section{INTRODUCTION}

Structures that have decoupled from the Hubble flow will show streaming motions that, while straightforward to detect as Doppler shifts along the line of sight, are difficult to distinguish from the Hubble expansion itself without an independent distance measure. Streaming motions across the line of sight (Nusser et al. 2012), or simply structures decoupled from the Hubble flow, however, are separable from the Hubble expansion because no proper motion will occur in a homogeneous expansion. Thus, with adequate astrometric precision, one can employ quasars as test masses to both detect structures that have decoupled from the Hubble flow (thus measuring masses) and to directly confirm the homogeneity of the Hubble expansion on large scales. If one can identify high brightness temperature light sources in fairly static structures, such as individual galaxies or galaxy clusters, then it is possible to obtain geometric distances and a measurement of the Hubble constant from observations of real-time recession.

The apparent size of "cosmic rulers" as a function of redshift is a canonical cosmological test, but the real-time change in the apparent size of such rulers caused by the cosmic expansion has not been explored. Here we examine the notion that gravitationally bound objects appear smaller as they recede, we develop the method by which this effect can be measured, and we apply this technique to extant proper motion data. We assume $H_{\circ}=72 \mathrm{~km} \mathrm{~s}^{-1} \mathrm{Mpc}^{-1}$ and a flat cosmology with $\Omega_{\Lambda}=0.73$ and $\Omega_{M}=0.27$.

\footnotetext{
${ }^{1}$ Center for Astrophysics and Space Astronomy, Department of Astrophysical and Planetary Sciences, University of Colorado, 389 UCB, Boulder, CO 80309-0389, USA; jdarling @ colorado.edu
}

\section{APPARENT PROPER MOTION OF COSMIC RULERS}

Given the definition of angular diameter distance, $\theta=\ell / D_{A}$, where a "ruler" of proper length $\ell$ subtends small angle $\theta$ at angular diameter distance $D_{A}$, both cosmic expansion and a changing $\ell$ can produce an observed fractional rate of change in $\theta$ :

$$
\frac{\Delta \theta / \Delta t_{\circ}}{\theta} \equiv \frac{\dot{\theta}}{\theta}=\frac{-\dot{D_{A}}}{D_{A}}+\frac{\dot{\ell}}{\ell}=\frac{-H(z)}{1+z}+\frac{\dot{\ell}}{\ell},
$$

where

$$
H(z)=H_{\circ} \sqrt{\Omega_{M, \circ}(1+z)^{3}+\Omega_{\Lambda}},
$$

$\Delta t_{\circ}$ is the observer's time increment, $\dot{\theta}$ is the proper motion, and $\dot{\ell}$ is the observed change in proper length, $\Delta \ell / \Delta t_{\circ}$, related to the physical (rest-frame) transverse velocity as $v_{\perp}=$ $\dot{\ell}(1+z)$. When the small-angle approximation is not valid, we assume that $D_{A}$ is the angular diameter distance to the midpoint of $\ell$ such that $\tan (\theta / 2)=(\ell / 2) / D_{A}$. For large angles, Equation (1) becomes

$$
\frac{\Delta \theta / \Delta t_{\circ}}{\sin \theta}=\frac{\dot{\theta}}{\sin \theta}=\frac{-H(z)}{1+z}+\frac{\dot{\ell}}{\ell} .
$$

All calculations use this exact relationship.

If $\ell$ is not a gravitationally influenced structure and grows with the expansion, then

$$
\frac{\dot{\ell}}{\ell}=\frac{H(z)}{1+z},
$$

exactly canceling the first term in Equation (1). In this case, $\dot{\theta}=0$, and there is no proper motion for objects co-moving with an isotropically expanding universe, as expected. If $\ell$ is 

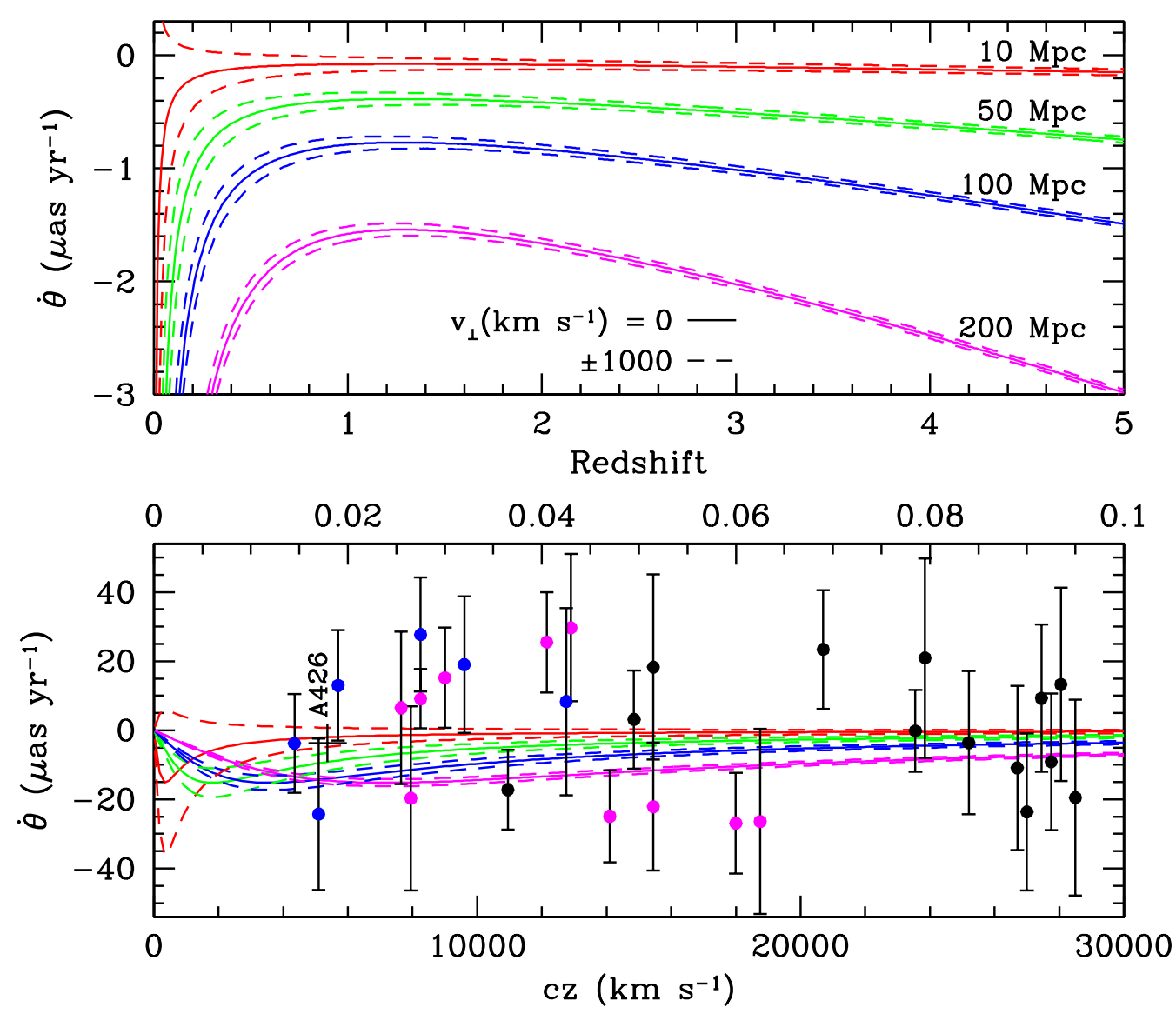

Figure 1. "Observer's plot" of the expected apparent recession of cosmic structures vs. redshift. The lower panel shows an expanded view of low redshifts. Red, green, blue, and magenta lines depict structures 10,50, 100, and $200 \mathrm{Mpc}$ in physical projected size. Solid and dashed lines indicate plane-of-sky rest-frame velocities $v_{\perp}=0$ and $\pm 1000 \mathrm{~km} \mathrm{~s}^{-1}$, respectively. The cross shows the expected effect for the nearby cluster Abell 426, the Perseus Cluster (Struble \& Rood 1999; Hamden et al. 2010). We assume that Abell 426 is completely decoupled from the Hubble expansion and static. In reality the apparent $\dot{\theta}$ would be modulated by Equation (12). Circles show measured values of the smallest-separation quasar pairs in the Titov et al. (2011b) sample with $|\dot{\theta}|<30 \mu$ as yr ${ }^{-1}$ and $\sigma_{\dot{\theta}}<30 \mu \mathrm{as} \mathrm{yr}^{-1}$ (Section 5, color-coded to match the projected size loci (black points have comoving separation 250-1500 Mpc). All points are consistent with $\dot{\theta}=0$ and none are inconsistent with the expected $\dot{\theta}$ for their separation (deviations are less than $3 \sigma$ ).

decoupled from the expansion, however, then for most reasonable gravitational motions, $\dot{\ell} / \ell$ is a minor modification to the expansion contribution to $\dot{\theta} / \theta$ because the expansion, except for small redshifts or small structures, dominates (Figures 1 and 2).

This "receding objects appear to shrink" observation does not rely on knowledge of the orientation or size of the "ruler" - any relative proper motion between objects that are coupled via gravity will allow a measurement of $\dot{\theta}$ because the measurement is differential.

Practically, this effect would be measured via the relative proper motion of high brightness temperature light sources such as quasars or masers. The convergence (or divergence) of a pair of test masses can be measured via

$$
\dot{\theta}_{12}=-\left(\boldsymbol{\mu}_{2} \cdot \hat{\theta}_{21}+\boldsymbol{\mu}_{1} \cdot \hat{\theta}_{12}\right)
$$

where $\boldsymbol{\mu}_{i}$ is the proper motion, and $\hat{\theta}_{12}$ and $\hat{\theta}_{21}$ are the unit vectors connecting the two test masses along a geodesic (the bearing from mass 1 to mass 2 and vice-versa). The angular separation of two points on a sphere, in terms of right ascension $(\alpha)$ and declination $(\delta)$, is

$$
\theta_{i j}=\arccos \left(\sin \delta_{i} \sin \delta_{j}+\cos \delta_{i} \cos \delta_{j} \cos \left[\alpha_{i}-\alpha_{j}\right]\right),
$$

and this separation changes with time as

$$
\begin{array}{r}
\dot{\theta}_{i j}=-\left(\cos \delta_{i} \sin \delta_{j}\left[\mu_{\delta, i}-\mu_{\delta, j} \cos \left(\alpha_{i}-\alpha_{j}\right)\right]\right. \\
+\sin \delta_{i} \cos \delta_{j}\left[\mu_{\delta, j}-\mu_{\delta, i} \cos \left(\alpha_{i}-\alpha_{j}\right)\right] \\
\left.-\cos \delta_{i} \cos \delta_{j} \sin \left(\alpha_{i}-\alpha_{j}\right)\left[\mu_{\alpha, i}-\mu_{\alpha, j}\right]\right) / \sin \theta_{i j}
\end{array}
$$

\section{INDIVIDUAL STRUCTURES: GEOMETRIC DISTANCE AND THE HUBBLE CONSTANT}

Individual galaxies or galaxy clusters will have a roughly fixed physical size over any human-timescale observation, so $\dot{\ell} \simeq 0$. For the local universe, $z \simeq 0, H(z) \simeq H_{\circ}$, and

$$
\dot{\theta} \simeq-H_{\circ} \theta \simeq-\theta\left(^{\circ}\right) 0.27 \mu{\mathrm{as} \mathrm{yr}^{-1}}^{-1}
$$

for small angles $\theta$. A large nearby cluster moving with the Hubble expansion, such as the Perseus Cluster (Abell 426), spanning $\sim 14^{\circ}$ (Hamden et al. 2010), would thus appear to shrink by $\sim 4 \mu \mathrm{as} \mathrm{yr}^{-1}$ (for comparison, the equivalent radial contraction velocity in a static universe would be $\sim 1400 \mathrm{~km} \mathrm{~s}^{-1}$ ). The Andromeda galaxy's $\sim 2^{\circ}$ molecular ring, approaching at effectively $5.3 H_{\circ}\left(-300 \mathrm{~km} \mathrm{~s}^{-1}\right.$ heliocentric at $780 \mathrm{kpc}$ ), will appear to grow by $\sim 3 \mu$ as $\mathrm{yr}^{-1}$ (equivalent to a radial expansion of $\sim 10 \mathrm{~km} \mathrm{~s}^{-1}$; Darling 

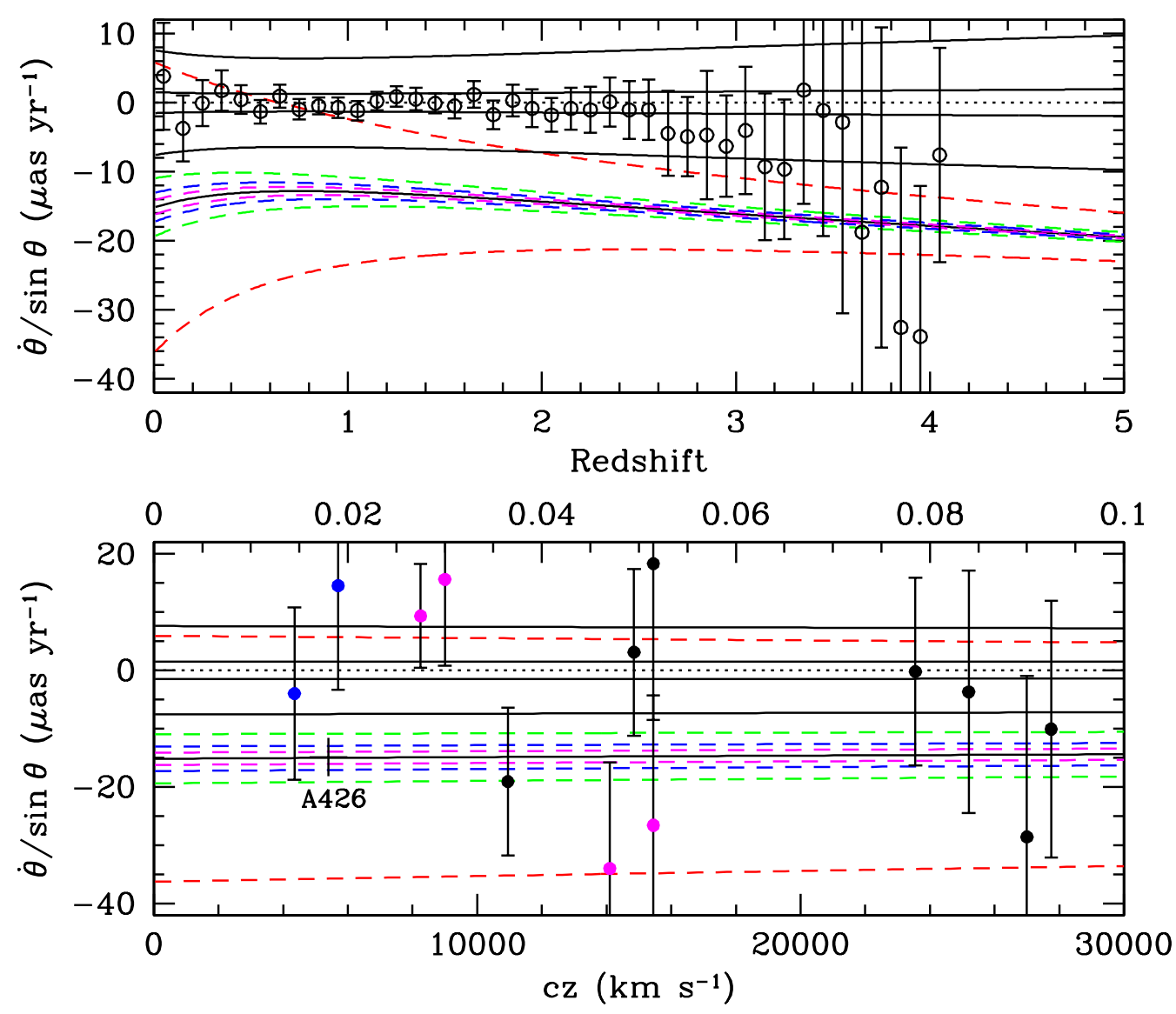

Figure 2. "Theorist's plot" of fractional apparent recession of cosmic structures vs. redshift. The lower panel shows an expanded view of low redshifts. Red, green, blue, and magenta lines depict plane-of-sky rest-frame velocities $v_{-}= \pm 1000 \mathrm{~km} \mathrm{~s}^{-1}$ for structures 10, 50, 100, and $200 \mathrm{Mpc}$ in physical projected size. The dotted black line shows pure Hubble expansion $(\xi=0$, Equation (12) $)$, and solid black lines indicate, from top to bottom, $\xi=-0.5,-0.1,+0.1,+0.5$, and +1 . The cross shows the expected effect for the nearby Perseus Cluster, Abell 426 (see the caption to Figure 1). Filled circles show measured values of the smallest-separation quasar pairs in the Titov et al. (2011b) sample with $|\dot{\theta}|<30 \mu \mathrm{as} \mathrm{yr}^{-1}$ and $\sigma_{\dot{\theta}}<30 \mu \mathrm{as} \mathrm{yr}^{-1}$ (Section 5), color-coded to match the projected size loci (black points have comoving separation 250-1500 Mpc). All points are consistent with $\dot{\theta}=0$ and none are inconsistent with the $\dot{\theta} / \sin \theta$ expected for their separation (deviations are less than $3 \sigma$ ). Open circles show a "null" test for all pairs, dominated by large-separation pairs ( $>1500 \mathrm{Mpc}$ ), which is consistent with pure Hubble expansion and excludes static structures, as expected, at $\sim 5-10 \sigma$ significance for $z \simeq 0.5-2$.

2011). While galaxies within clusters and individual maseremitting regions within galaxies may exhibit peculiar velocities, a virialized cluster or a rotating disk galaxy will not exhibit a radial change in size that could be confused with the cosmological recession (see also Figure 1). A large peculiar motion such as the initial infall expected for the Bullet Cluster, however, with $v_{\perp}=3000 \mathrm{~km} \mathrm{~s}^{-1}$ and $\ell=5 \mathrm{Mpc}$ at $z=0.296$ (Mastropietro \& Burkert 2008), would produce

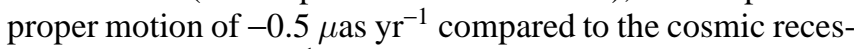

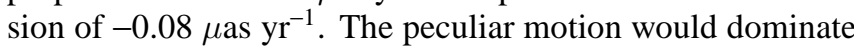
the proper motion in this case because the pre-Bullet Cluster had a small size, large peculiar motion, and thus large $\dot{\ell} / \ell$.

The apparent shrinking of receding objects provides a direct geometric measurement of the Hubble constant $H_{\circ}$ (modulo peculiar velocity),

$$
H_{\circ} \simeq-\frac{\dot{\theta}}{\theta},
$$

and the geometric (proper) distance,

$$
D \simeq-v \frac{\theta}{\dot{\theta}},
$$

which is peculiar velocity-independent (the exact relationship is modulated by the ratio between proper distance (Hubble's
Law) and angular size distance, $1+z$, but these are very similar at $z \simeq 0$ ). $\theta, \dot{\theta}$, and Doppler velocity $v$ are observable quantities: this implies that the Hubble constant and distance can be directly measured from the apparent proper motion of receding objects. These measurements do not rely on any information about the physical size or orientation of the observed shrinking object, in contrast to the canonical cosmological "standard ruler" tests. Moreover, since the Doppler velocity and the angular size can be measured extremely precisely, the uncertainty in these measurements is dominated by the uncertainty in $\dot{\theta}$. And while the measurement of $H_{\circ}$ relies on an assumption of motion entrained in the Hubble flow (small peculiar velocity), the measurement of geometric distance does not rely on any assumptions because receding objects appear to shrink regardless of the reason for the recession (peculiar or cosmological).

Measuring apparent proper motions requires compact luminous (high brightness temperature) sources at the boundaries of the receding object or structure. Typically these sources will be masers, which are severely distance-limited, or active galactic nuclei (AGNs). In the case of galaxy clusters, bright AGNs on the periphery of clusters are rare; they typically reside in cluster centers. Since quasars and galaxy clusters mark density peaks in large scale structure, it stands to reason that 

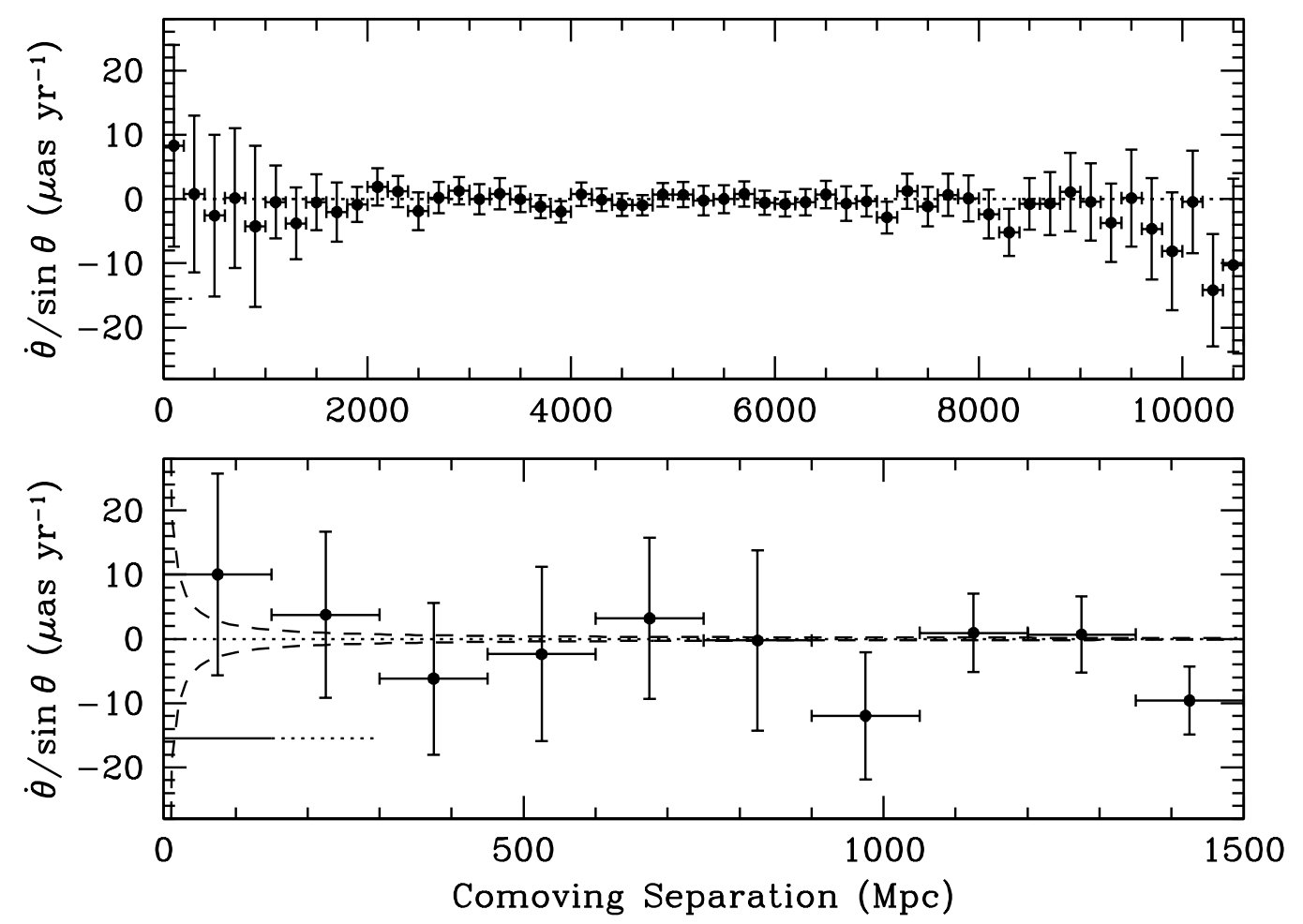

Figure 3. Measured pairwise divergence or convergence vs. comoving separation of radio sources in the Titov et al. (2011b) proper motion sample. Top: pairwise fractional proper motion of large-separation pairs showing no systematic or significant offset from pure Hubble expansion. Bottom: pairwise fractional

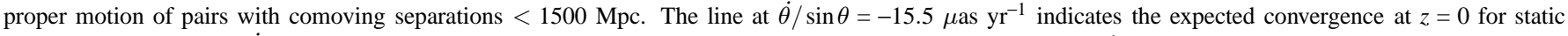
structures (Equation 3, $\dot{\ell}=0$ ). The dashed lines show the effect of a peculiar transverse motion of $\pm 1000 \mathrm{~km} \mathrm{~s}^{-1}$ on otherwise comoving pairs. The point with comoving separation less than $150 \mathrm{Mpc}$ is consistent with Hubble expansion and static structures (and is therefore inconclusive given the present sample size and measurement precision).

gravitationally bound or Hubble flow-decoupled structures as revealed by quasars and clusters of galaxies will show a relative proper motion as large scale structure decouples from the universal expansion.

\section{APPARENT PROPER MOTION OF LARGE SCALE STRUCTURES}

Separating $\dot{\ell} / \ell$ into expansion and peculiar velocity parameterized by $\xi$, which can be related to density contrast $\delta \rho /\langle\rho\rangle$ in the linear regime but is otherwise a free parameter for peculiar velocity scaled to the local Hubble expansion,

$$
\frac{\dot{\ell}}{\ell}=\frac{H(z)}{1+z}(1-\xi) \text {. }
$$

Equation (1) becomes

$$
\frac{\dot{\theta}}{\theta}=\frac{-H(z)}{1+z} \xi .
$$

Thus, if $\xi=0$ (i.e., $\delta \rho=0$ ), the structure expands with the Hubble flow and there is no apparent proper motion. If $\xi=1$, $\dot{\ell}=0$, and $\ell$ is a cosmic ruler. If $\xi>0$, there is apparent convergence, and if $\xi<0$, there is apparent divergence (i.e., voids). For quasar pairs, $\delta \rho /\langle\rho\rangle$ is usually in the linear regime (typical quasar pairs are close to or much farther apart than the homogeneity scale). Objects at the edges of voids are expected to move apart due to collapse away from voids, which are otherwise expanding with the Hubble flow, so a slight divergence could be expected. This would be a few $\mu$ as at most at low redshift and $<1 \mu$ as $\mathrm{yr}^{-1}$ at $z \gtrsim 0.1$.

Figure 1 shows the "observer's plot" of the expected proper motion of structures of various size scales and peculiar plane- of-sky velocities versus redshift along with measurements of individual quasar pairs (Section [5). Peculiar motions are a small contribution to $\dot{\theta}$ except for small or nearby structures, although for large structures the quantity of relevance is the velocity gradient. For example, the Great Wall's $-15 \mu \mathrm{as} \mathrm{yr}^{-1}$ recession-equivalent contraction velocity is $\sim 9000 \mathrm{~km} \mathrm{~s}^{-1}$, which is a velocity gradient of only about $37 \mathrm{~km} \mathrm{~s}^{-1} \mathrm{Mpc}^{-1}$. In any case, structures, with the exception of voids, do not generally experience peculiar expansion, so gravitational contraction enhances the proper motion signal, and the dominant consideration becomes the impact of $\xi$ on apparent contraction.

Figure 2 shows the "theorist's plot" of the expected fractional proper motion for various $\xi$ values and peculiar planeof-sky velocities versus redshift along with measurements of individual and binned quasar pairs (Section 5). $H(z) /(1+z)$ is a slowly varying function of redshift and can be approximated as a constant, $\sim 15 \mu$ as $\mathrm{yr}^{-1}$. This plot properly shows the enhanced effect of $v_{\perp}$ on smaller structures and demonstrates that small-angular-separation quasar pairs with precisely measured $\dot{\theta}$ are needed to make the first measurement of the cosmic recession effect.

\section{A FIRST APPLICATION OF DATA}

We employ the Titov et al. (2011b) proper motion measurements of 555 radio sources, using the 507 with redshifts (Titov et al. 2011a, updated online catalog), to attempt a first test of the expected real-time convergence of Hubble flowdecoupled pairs and to confirm the pure Hubble expansion of large-separation pairs. The data were obtained from 5030 

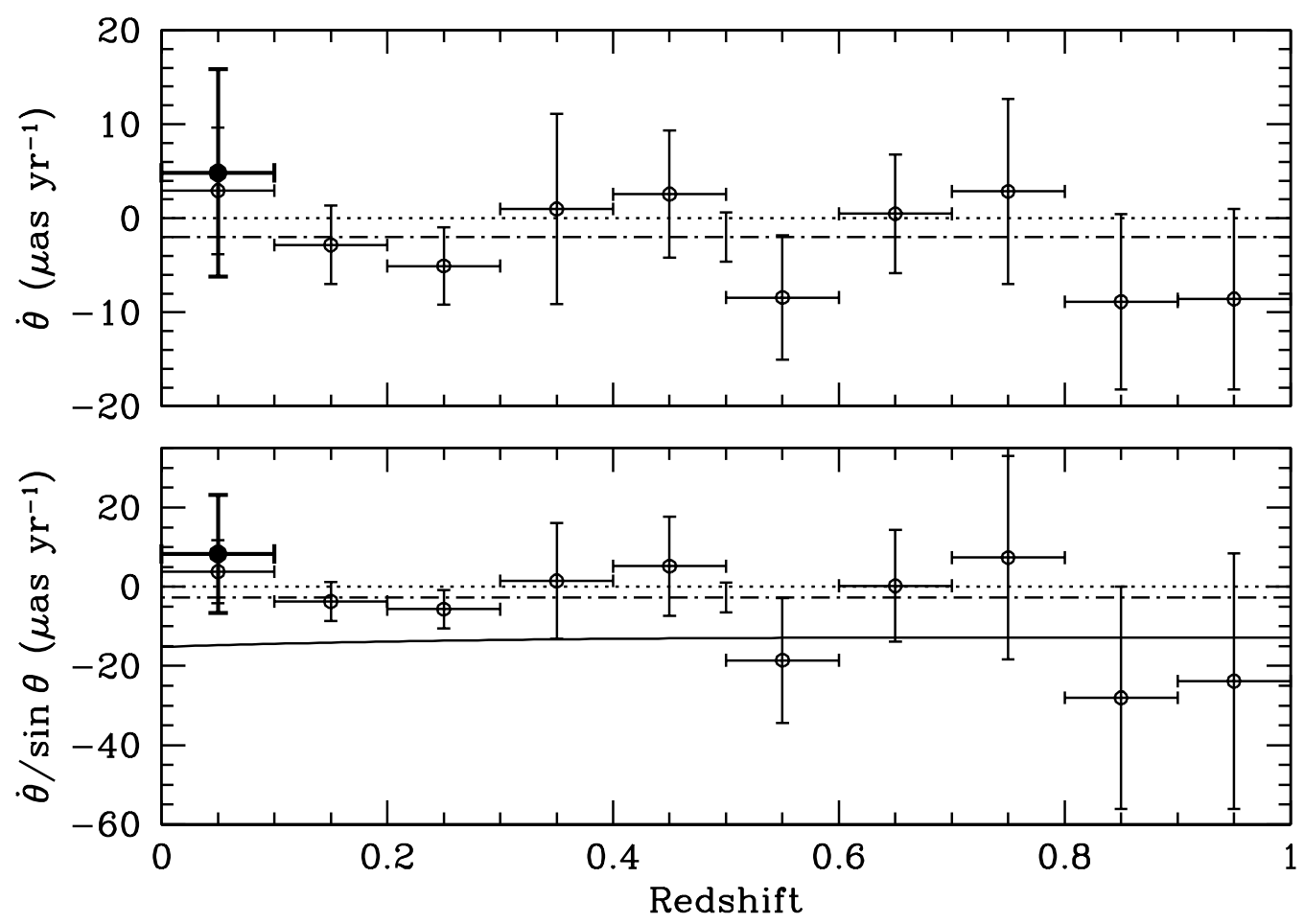

Figure 4. Measured pairwise divergence or convergence vs. redshift of radio sources in the Titov et al. (2011b) proper motion sample. Bold filled circles indicate pairs with comoving separation less than $200 \mathrm{Mpc}$, and empty circles indicate pairs with comoving separation between $200 \mathrm{Mpc}$ and $1500 \mathrm{Mpc}$ (Figure 2 shows all pairs and redshifts). Top: pairwise proper motion. Bottom: pairwise fractional proper motion. The solid line indicates the expected convergence for static structures, $\dot{\theta} / \sin \theta=-H(z) /(1+z)$ (Equation [3, $\dot{\ell}=0)$. Dot-dash lines show the mean of all large comoving separation pairs $(200-1500 \mathrm{Mpc})$ in $0<z<1$, and the small error bars at $z=0.5$ show the uncertainty in the mean, demonstrating that the large-separation pairs are consistent with pure Hubble expansion and inconsistent with static structures with $3.5 \sigma$ confidence. The single point with comoving separation less than 200 Mpc, however, is consistent with Hubble expansion and with the expectation for static structures.

sessions of the permanent geodetic and astrometric very long baseline interferometry (VLBI) program, which includes the Very Long Baseline Array at $8.4 \mathrm{GHz}$ in 1990-2010 using a relaxed per-session no-net rotation constraint (Titov et al. 2011b).

While pairwise proper motions are minimally affected by the secular aberration drift caused by the barycenter acceleration about the Galactic center, we nonetheless subtract the dipole proper motion pattern first identified by Titov et al. (2011b) and confirmed by Xu et al. (2012) but employing the Reid (2013) results, $R_{0}=8.38 \pm 0.18 \mathrm{kpc}$ and $\Theta_{0}=243 \pm$ $7 \mathrm{~km} \mathrm{~s}^{-1}$, which give a dipole amplitude of $5.0 \pm 0.3 \mu \mathrm{as} \mathrm{yr}^{-1}$, from the observed proper motion vector field. We assume that the acceleration direction is exactly toward the Galactic center and do not include the out-of-the-disk acceleration described by Xu et al. (2012) in our correction.

In order to omit poorly-measured proper motions and objects with large intrinsic proper motions, objects used in this analysis are restricted to proper motion and uncertainty

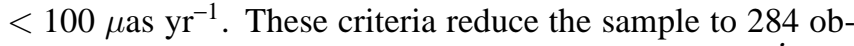
jects. Pairs of objects are likewise restricted to have $|\dot{\theta}|$ and $\sigma_{\dot{\theta}}<100 \mu$ as $\mathrm{yr}^{-1}$. While these choices are somewhat arbitrary, different cutoff values of the same order of magnitude have minor impact on the results.

The individual pairs with comoving separation $<150 \mathrm{Mpc}$ and small $\dot{\theta}$ in Figure 1 are ICRF $\mathrm{J} 110427.3+381231 \quad(z=0.03)$ and $\mathrm{J} 123049.4+122328$

\footnotetext{
2 The National Radio Astronomy Observatory is a facility of the National Science Foundation operated under cooperative agreement by Associated Universities, Inc.
}

$(z=0.004), \quad \mathrm{J} 110427.3+381231$ and $\mathrm{J} 163231.9+823216$ $(z=0.025), \quad \mathrm{J} 110427.3+381231$ and $\mathrm{J} 165352.2+394536$ $(z=0.034), \mathrm{J} 165352.2+394536$ and $\mathrm{J} 180650.6+694928$ $(z=0.051), \mathrm{J} 123049.4+122328$ and $\mathrm{J} 163231.9+823216$, and $\mathrm{J} 123049.4+122328$ and $\mathrm{J} 165352.2+394536$. The latter two pairs also have small $\dot{\theta} / \sin \theta$ (Figure 2).

Figure 3 shows the divergence/convergence of radio source pairs (Equations (5) and (7)) versus their comoving separation. The comoving separation is calculated from comoving proper distances using the cosine rule and Equation (6):

$$
r_{i j}^{2}=r_{i}^{2}+r_{j}^{2}-2 r_{i} r_{j} \cos \theta_{i j} \text {. }
$$

Error bars are estimated from bootstrap resampling. The expected signal for static structures $(\dot{\ell}=0)$ at $z=0$ is $\dot{\theta} / \sin \theta=$ -15.5 $\mu$ as $\mathrm{yr}^{-1}$ (Equation (3)). The data point with comoving separation $<150 \mathrm{Mpc}$ is consistent with this value $\left(\dot{\theta} / \sin \theta=+10 \pm 16 \mu\right.$ as $\mathrm{yr}^{-1} ; 1.6 \sigma$ separation $)$, but it is also consistent with pure Hubble expansion $(\dot{\theta}=0)$; more pairs or precision are needed. Pairs with comoving separations 150-1500 Mpc are consistent with pure Hubble expansion, as expected, as are those with separations 0-1500 Mpc, which are inconsistent with the signal from static structures at $z=0$ with $3.7 \sigma$ significance: $\langle\dot{\theta} / \sin \theta\rangle=-2.3 \pm 3.6 \mu$ as $\mathrm{yr}^{-1}$ and

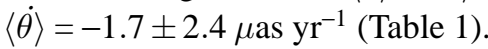

Figure 4 shows the divergence/convergence of pairs versus their mean redshift, grouped into two populations: those with comoving separations less than $200 \mathrm{Mpc}$, and those with comoving separations between 200 and $1500 \mathrm{Mpc}$ (Table 1, Figure 2] shows all pairs). Because the bright radio sources suitable for proper motion measurements have a low 
Table 1

Binned Pairwise Proper Motions

\begin{tabular}{|c|c|c|c|}
\hline $\begin{array}{l}\text { Comoving } \\
\text { Separation } \\
(\mathrm{Mpc})\end{array}$ & $\langle z\rangle$ & $\begin{array}{c}\langle\dot{\theta}\rangle \\
\left(\mu \mathrm{as}^{-1} \mathrm{r}^{-1}\right)\end{array}$ & 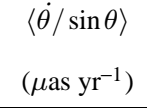 \\
\hline 0-200 & $0.0-0.1$ & $4.8(11.0)$ & $8.3(14.9)$ \\
\hline $200-1500$ & $0.0-0.1$ & $2.9(6.7)$ & $3.8(7.9)$ \\
\hline $200-1500$ & $0.1-0.2$ & $-2.8(4.2)$ & $-3.7(5.0)$ \\
\hline $200-1500$ & $0.2-0.3$ & $-5.1(4.1)$ & $-5.6(4.8)$ \\
\hline $200-1500$ & $0.3-0.4$ & $1.0(10.1)$ & $1.5(14.7)$ \\
\hline $200-1500$ & $0.4-0.5$ & $2.6(6.8)$ & $5.2(12.5)$ \\
\hline $200-1500$ & $0.5-0.6$ & $-8.4(6.6)$ & $-18.6(15.8)$ \\
\hline $200-1500$ & $0.6-0.7$ & $0.5(6.3)$ & $0.2(14.1)$ \\
\hline $200-1500$ & $0.7-0.8$ & $2.9(9.8)$ & $7.4(25.7)$ \\
\hline $200-1500$ & $0.8-0.9$ & $-8.9(9.3)$ & $-28.1(28.1)$ \\
\hline $200-1500$ & $0.9-1.0$ & $-8.6(9.6)$ & $-23.8(32.3)$ \\
\hline $200-1500$ & $0.0-1.0$ & $-2.0(2.6)$ & $-2.7(3.7)$ \\
\hline 0-150 & Any & $6.7(8.9)$ & $10.0(15.7)$ \\
\hline $150-300$ & Any & $2.1(11.2)$ & $3.8(12.9)$ \\
\hline $300-450$ & Any & $-3.3(7.4)$ & $-6.2(11.8)$ \\
\hline $450-600$ & Any & $-5.0(8.2)$ & $-2.4(13.6)$ \\
\hline $600-750$ & Any & $0.4(6.8)$ & $3.2(12.6)$ \\
\hline $750-900$ & Any & $2.7(7.5)$ & $-0.2(14.0)$ \\
\hline $900-1050$ & Any & $-6.9(5.6)$ & $-12.0(9.9)$ \\
\hline $1050-1200$ & Any & $0.9(4.5)$ & $0.9(6.1)$ \\
\hline $1200-1350$ & Any & $-0.0(4.5)$ & $0.7(5.9)$ \\
\hline $1350-1500$ & Any & $-6.1(3.5)$ & $-9.6(5.3)$ \\
\hline $0-1500$ & Any & $-1.7(2.4)$ & $-2.3(3.6)$ \\
\hline Any & Any & $-0.35(0.52)$ & $-0.36(0.62)$ \\
\hline
\end{tabular}

Note. - Bold entries indicate comoving separations where proper motions are expected to deviate from pure Hubble flow. Parenthetical values are $1 \sigma$ uncertainties.

areal density, the small-separation pairs are necessarily at low redshift $(z<0.1)$ where large angles can span small proper distances. For large-separation pairs, the redshift difference between pairs can be larger than the $\Delta z=0.1$ redshift bin, and binned redshifts are averages, not the redshifts corresponding to averaged distances. Static structures should show

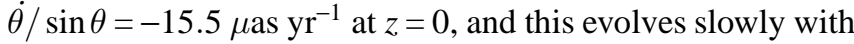
redshift. The sole data point with small comoving separation $(<200 \mathrm{Mpc})$ is consistent with this value, $\dot{\theta} / \sin \theta=+8.3 \pm$

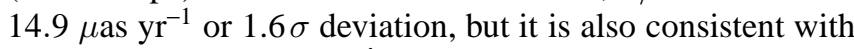
pure Hubble expansion, $\dot{\theta} / \sin \theta=0$. More small-separation pairs or precision are needed. Pairs with comoving separations $200-1500 \mathrm{Mpc}$ at $z<1$ are consistent with pure Hubble

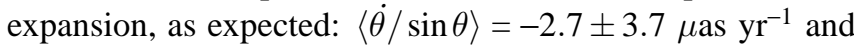

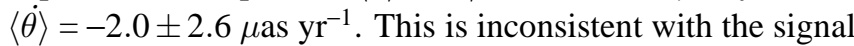
from static structures at $z=0$ at $3.5 \sigma$ significance.

Using all pairs and all redshifts (Figure 2), we reject the static locus at $\sim 5-10 \sigma$ significance for $z \simeq 0.5-2$. Likewise, for the entire sample unbinned in redshift we obtain a "null test" pairwise proper motion of $\langle\dot{\theta} / \sin \theta\rangle=-0.36 \pm$ $0.62 \mu \mathrm{as} \mathrm{yr}^{-1}$ and $\langle\dot{\theta}\rangle=-0.35 \pm 0.52 \mu \mathrm{as} \mathrm{yr}^{-1}$, consistent with pure Hubble expansion (the negligible difference between $\dot{\theta}$ and $\dot{\theta} / \sin \theta$ is due to the angular separation averaging to $90^{\circ}$; the two values no longer match if one does not subtract the aberration drift signature from the proper motion vector field). These precise measurements are possible despite the large apparent proper motions intrinsic to radio jets because intrinsic motions are not correlated between objects.

\section{DISCUSSION}

As Figures 3 and 4 show, the expected pairwise convergence effect should be detectable using current angular resolution, astrometry, and proper motion sensitivity. The major impediment to progress is the limited number of close quasar pairs. The binned large-separation pairs can reach uncertainties of $\sim 1 \mu \mathrm{as} \mathrm{yr}^{-1}$, which is more than adequate to detect convergence and recession of structures were similar numbers of sub-150 Mpc pairs observed. Higher bandwidth VLBI recording can grow the radio proper motion sample by an order or magnitude, but the large intrinsic proper motions manifested in many radio sources will still be a limitation. Optical proper motions obtained by the Gaia mission ${ }^{3}$ will benefit from a vastly larger sample of $\sim 500,000$ quasars and from negligible intrinsic proper motion. Gaia will achieve astrometry of $\sim 80 \mu$ as for $V=18$ mag stars (de Bruijne et al. 2005).

Future observations, whether radio or optical, should be able to detect the statistical convergence signal and may detect the recession effect in single nearby pairs as well. Individual low-redshift pairs in the (Titov et al.2011b) are sample already within a factor of a few of the precision needed to test the recession effect (Figure 2), and the geodetic observations were not designed for this purpose. A true "moving cluster" observation of a galaxy cluster may someday be possible, providing a geometric distance from cosmic expansion alone.

\section{CONCLUSIONS}

While the sample of small-separation quasar pairs with precise proper motion measurements is as-yet too sparse to detect the cosmic recession and collapse of structure, largeseparation test masses have now been measured with high significance to be comoving with the Hubble expansion and can serve as a reference frame for small-separation pairs that will significantly deviate from the Hubble flow due to gravity. This relative measurement of small-separation versus largeseparation quasar pairs will mitigate possible systematic effects inherent in such precise proper motion measurements given the large intrinsic proper motions seen in radio sources. Improved VLBI astrometry and the Gaia astrometry mission will likely detect the departure of structures from pure Hubble expansion in a statistical sample as well as for individual structures. It may also be possible to obtain geometric distances and measure the Hubble constant by observing relatively static objects such as individual galaxies or galaxy clusters.

The author thanks Wright (2006) for the online cosmology calculator and the anonymous referee for helpful comments. This research has made use of the NASA/IPAC Extragalactic Database (NED) which is operated by the Jet Propulsion Laboratory, California Institute of Technology, under contract with NASA.

\section{REFERENCES}

Darling, J. 2011, ApJ, 732, L2

de Bruijne, J., Perryman, M., Lindegren, L., Jordi, C., Høg, E., Katz, D., \& Cropper, M. 2005, Gaia-JdB-022 Technical Note

Hamden, E. T., Simpson, C. M., Johnston, K. V., \& Lee, D. M. 2010, ApJ, 716, L205

Mastropietro, C. \& Burkert, A. 2008, MNRAS, 389, 967

Nusser, A., Branchini, E., \& Davis, M. 2012, ApJ, 755, 58

3 http://www.rssd.esa.int/SYS/docs/ll_transfers/project=PUBDB/\&id=448635.pdf 
Reid, M. J. 2013, in IAU Symp. 289, Advancing the Physics of Cosmic Distances, ed. R. de Grijs (Cambridge: Cambridge Univ. Press), 188 Struble, M. F. \& Rood, H. J. 1999, ApJS, 125, 35
Titov, O., Jauncey, D. L, Johnston, H. M., Hunstead, R., W., \& Christensen, L. 2011a, AJ, 142, 165

Titov, O., Lambert, S. B., \& Gontier, A.-M. 2011b, A\&A, 529, A91

Wright, E. L. 2006, PASP, 118, 1711

Xu, M. H., Wang, G. L., \& Zhao, M. 2012, A\&A, 544, A135 